\title{
Neonatal group B streptococcal infection in South Bedfordshire, 1993-1998
}

\author{
Kathryn Beardsall, M H Thompson, R J Mulla
}

\begin{abstract}
Background-Group B streptococcus (GBS) is now the leading cause of neonatal bacterial sepsis in the western world. The incidence of GBS infection in the United States has been determined, and guidelines produced and implemented for the prevention of neonatal infection. Neither incidence nor guidelines are currently established in the United Kingdom.

Aim-To define the pattern of neonatal infection within one hospital (Luton and Dunstable Hospital).

Method-A six year retrospective analysis was performed.

Result-An incidence of early onset GBS of 1.15 per 1000 deliveries, comparable with that documented in the United States, was found.

(Arch Dis Child Fetal Neonatal Ed 2000;82:F205-F207)
\end{abstract}

Keywords: group B streptococcus; sepsis; neonatal; morbidity

Group B streptococcus (GBS) is now the most important cause of bacterial sepsis in the newborn, with a reported incidence of $0.5-2$ per 1000 live births, ${ }^{1}$ and causing significant morbidity and mortality. Its importance has recently been reviewed. ${ }^{2}{ }^{3}$ Neonatal infection is of either early or late onset, defined by development of symptoms before or after the first week of life. Risk factors have been identified that increase the probability of neonatal infection, and it now seems clear that, if intrapartum antibiotics are given to women with risk factors, vertical transmission of GBS can be considerably reduced. ${ }^{4}$ Few data are available about the prevalence of the disease in the United Kingdom. We set out to look at the extent of the disease in our hospital where no prevention policy existed.

Department of

Paediatrics, Luton and Dunstable Hospital, Lewsey Road, Luton, Bedfordshire, UK K Beardsall

M H Thompson

Public Health Laboratory Luton R J Mulla

Correspondence to: Dr Beardsall, Neonatal Unit, Obstetric Hospita University College Hospital, Huntley Street, London WC1E 6A, UK

email:

kbeardsall@btinternet.com

Accepted 15 September 1999

\section{Methods}

The period studied was between January 1993 and December 1998, based at the Luton and Dunstable Hospital, a subregional referral centre within a multiracial community. The neonatal delivery rate varied from 3728 to 4389 a year, with a total of 24267 live births. GBS infected cases were defined as neonates with blood or cerebrospinal fluid (CSF) cultures positive for GBS. These cases were identified from a microbiology database, and then both neonatal and maternal notes were examined. The obstetric department previously had no defined policy for screening or treatment of women at risk of GBS in pregnancy or labour.
Blood cultures had been taken from neonates with clinical signs of infection. Culture bottles were then incubated at $37^{\circ} \mathrm{C}$ for five to seven days using a Sentinel 4-Drawer system (Difco Ltd, Franklin Lakes, New Jersey, USA) until March 1996, from which time the BacTAlert microbial detection system (Organon Teknika Corp, Boxtel, the Netherlands) was used. There is no evidence to suggest a difference in sensitivities to growing GBS between these two systems. Positive isolates were confirmed by antigen testing using a streptococcal grouping kit (Oxoid Ltd, Basingstoke, Hampshire, UK).

We compared our cases with data available from the annual maternity figures published in the regional maternity database for characteristics such as ethnicity, maternal age, and parity. We considered significant prenatal risk factors for neonatal GBS infection to be any of the following: previously infected infant; GBS urinary tract infection or localised from maternal swabs (although there was no routine screening); prematurity ( $<37$ weeks gestation); prolonged rupture of membranes ( $>18$ hours); maternal temperature $\left(>38^{\circ} \mathrm{C}\right)$.

To assess level of deprivation within geographical areas, we used the Public Health Common Data set (compiled by the Department of Health), and used the underprivileged area score (Jarman scores) for health authorities. We then used maternal addresses to give our cases a Jarman score dependent on their electoral ward. A positive Jarman score is an indicator of an area of deprivation; the more positive a value the more deprived that area is compared with one with a smaller or negative value.

\section{Results}

We identified 34 cases of neonatal GBS infection, an incidence of 1.4 per 1000 live births $(95 \%$ confidence interval (CI) 0.98 to 1.88). In five of these cases, GBS also grew from CSF cultures. Twenty eight cases were classified as early onset. This gives an incidence of early onset GBS disease of 1.15 per 1000 live births (95\% CI 0.64 to 1.66$)$. There was an increasing incidence of early onset disease over the six years studied (fig 1).

EARLY ONSET DISEASE

It was found that in $32 \%$ of cases, birth was at $<37$ weeks gestation, a higher proportion than that of the general neonatal population $(8.6 \%)$. Although the risk of infection is significantly higher in premature neonates, most $(58 \%)$ of the infected neonates were born at term. There was a high proportion of primiparous mothers: 


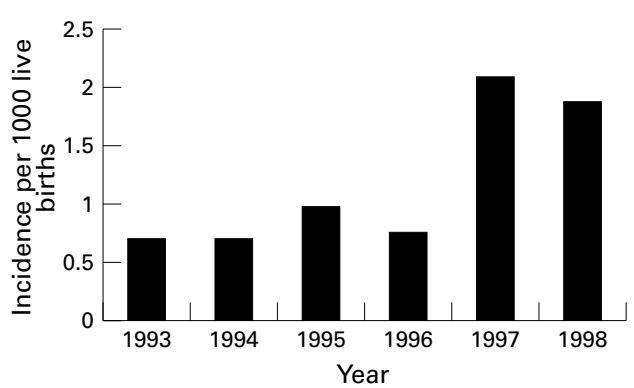

Figure 1 Incidence of group B streptococcal infection at Luton and Dunstable Hospital 1993-1998. Values are mean (SEM). Significant increase in incidence over time, $\chi^{2}$ test of trend $p=0.028$.

$61 \%$ compared with the hospital maternity population of $41 \%$. There was no association with age less than 20 years or ethnic origin. No mothers had had a previously infected infant or positive swabs or bacteriuria with GBS during this pregnancy. There was prolonged rupture of membranes in 13 cases and high maternal temperature in five.

In 18 cases there was a risk factor present during labour; of these, only four mothers received any antibiotics before delivery and none more than four hours before delivery. In only 10 cases were there no risk factors present before delivery. Only one infant presented more than 24 hours after delivery; this was the only early onset case for which a positive CSF culture for GBS was found. Six of the neonates spent more than two weeks in hospital; the average length of stay was 12.5 days. Four infants died; all were premature $(24,24+6$ days, 26, 28 weeks gestation). Currently at preliminary follow up there is concern about possible hypertonia in two cases.

\section{LATE ONSET DISEASE}

There were six cases of late onset disease; one of these infants was born at term and the other five at less than 34 weeks gestation. In two of these cases, there had been prolonged rupture of membranes before delivery, and, in one, the mother had also been pyrexial. In four of these cases, positive CSF cultures were obtained, and there was significant morbidity and mortality after late onset disease. One died in the neonatal period, three have severe neurological impairment, and only two remain well (at 9 months and 3 years).

\section{Discussion}

This study reports an incidence of early onset GBS of 1.15 per 1000 live births as identified by positive blood cultures. This figure is more than double that detected in a recent study from Oxford ${ }^{1}$ and higher than that reported in many areas of the United States. In contrast with the study from Oxford, we found a significant increase in incidence over time $(\mathrm{p}=0.028$ using $\chi^{2}$ test for trend).

The community is likely to be quite different in these two areas both racially and socioeconomically. Studies in North America have shown differences in GBS colonisation rates between ethnic groups within one geographical area. Our numbers were too small to show a

\section{Key messages}

- An incidence of early onset GBS infection of 1.15 per 1000 live births has been shown, with increasing incidence over the six year period studied

- The occurrence of late onset, as well as early onset, disease is associated with the presence of risk factors at the time of delivery

- This high prevalence of disease in the population served by the Luton and Dunstable Hospital suggests that a screening or risk factor based approach to prevention would be cost effective

significant difference between racial groups, but the relatively high prevalence of GBS infection in this study may be a consequence of relatively poor socioeconomic status. The average Jarman score for our neonatal cases was +19.14 , whereas the score for Bedfordshire is -7.47 .

By using only microbiologically proven cases to define the infected population, we will not have included a number of neonates with a clinical diagnosis of GBS infection, but with sterile blood cultures. Therefore the "true" prevalence of GBS infections may be considerably higher than we report.

This study reports significant morbidity and mortality following late onset disease, with severe neurological sequelae in $50 \%$ of cases. There is little published information available about the role of intrapartum risk factors or timing of colonisation in infants who develop late onset disease. It is interesting to note, however, that, in five out of six cases of late onset disease, birth was premature, in two there was prolonged rupture of the membranes, and in one case the mother was pyrexial during delivery. Colonisation therefore may have occurred at the time of delivery.

The incidence of GBS infection in the United States has been determined, and guidelines produced and implemented for the prevention of neonatal infection. ${ }^{5}$ Neither incidence nor guidelines are currently established in the United Kingdom. Our study clearly shows a significant incidence of early onset GBS infection and suggests an increasing incidence over time. An active intervention policy therefore seems warranted in our area. In a climate of increasing emphasis on preventive medicine, and medical litigation, it is worth considering the implications of not developing a health care policy for the prevention of such a consequential cause of perinatal morbidity and mortality. More information is needed about the prevalence and pattern of disease in the United Kingdom so that an appropriate plan for intervention can be made that reflects the pattern of disease within a local area.

We are grateful to Isabel Dodd for help in obtaining data from the Luton and Dunstable computer database, Ann Martin for retrieval of clinical notes for both mothers and infants, Sarah Shore for help with statistical analysis, and Dr Stephen W Shore for help with statistical
Davies for helpful comments. 
1 Moses LM, Heath PT, Wilkinson AR, Jeffrey HE, Isaacs D. Early onset group B streptococcal neonatal infection in 98;79:F148-9

2 Siegel JD. Prophylaxis for neonatal group B streptococcus infections. Semin Perinatol 1998; 22:33-49.

3 McKenna DS, Iams JD. Group B streptococcal infections. Semin Perinatol 1998;22:267-76.

\section{Commentary}

Group B streptococci (GBS) are an important cause of disease in newborn infants. Experience in the United States and Australia suggests that the incidence of early onset disease may be reduced by administration of antibiotics during labour; policies for identifying newborns at risk use either a screening method to detect maternal genital carriage or a clinical approach based on other known risk factors. ${ }^{12}$ However, the important questions raised by David Isaacs in 1998 for the United Kingdom $^{3}$ remain to be answered. Local studies in the United Kingdom show different minimum estimates of early onset GBS infection: 0.5 per 1000 live births in Oxfordshire, ${ }^{4}$ 0.8 in South West England, ${ }^{5} 0.95$ in Sunderland, ${ }^{6}$ and, in this paper, an estimate of 1.15 in Bedfordshire. If these estimates are combined, an incidence of 0.72 per 1000 live births (95\% CI $0.60-0.85)$ is yielded. This figure is similar to the incidence above, for which a risk factor approach to prevention becomes cost beneficial in the United States (0.6 per 1000), but below that for which universal maternal screening for GBS has been recommended (1.2 per 1000). ${ }^{3}$ Where risk factors have been investigated in the United Kingdom, they differ somewhat from patterns in the United States, with young maternal age and ethnic minority group membership not being over represented. A major weakness is that studies in the United Kingdom have rarely included major urban and deprived areas. It is difficult to proceed further with policy development in the United Kingdom without gathering data from larger populations and
4 Centers for Disease Control. Adoption of hospital policies for prevention of perinatal group B streptococcal disease, 1998;47:665-70.

5 Centers For Disease Control. Prevention of perinatal group B streptococcal disease: a public health perspective. MMWR CDC Surveill Summ 1996;45(RR-7):124

undertaking health economic analyses based on United Kingdom data. An enhanced laboratory based surveillance study is now underway in several large London hospitals, and a year long national surveillance study involving paediatricians, microbiologists, and consultants in communicable disease control is being conducted through the British Paediatric Surveillance Unit. This study will need to incorporate data on local antibiotic practices, as increasingly these are being instituted. Related case-control studies are also required to identify risk factors, and an economic analysis specific for the United Kingdom must be carried out before any risk factor or microbiological screening procedure can formally be supported.

ANGUS NICOLL

PHLS Communicable Disease Surveillance Centre

St George's Hospital Medical School

PAUL HEATH

1 Centers for Disease Control. Adoption of hospital policies or prevention of perinatal group B streptococcal disease, United States, 1997. MMWR CDC Surveill Summ 1998;47:665-70

2 Isaacs D, Royle JA for the Australian Study Group for Neonatal Infections. Intrapartum antibiotics and early onset neonatal sepsis caused by group B Streptococcus and by other organisms in Australia. Pediatr Infect Dis $\mathcal{f}$ 1999;18:524-8.

3 Isaacs D. Prevention of early onset group B streptococcal disease: screen, treat or observe? Arch Dis Child Fetal Neonatal Ed 1998;79:F81-2.

4 Moses LM, Heath PT, Wilkinson AR, Jeffery HE, Isaacs D. Early onset group B streptococcal neonatal infection in Oxford 1985-1996. Arch Dis Child Fetal Neonatal Ed 1998;79:F148-9

5 Fey RJ, Stuart J, George R. Neonatal group B streptococcal disease in England and Wales 1981-1997 [abstract]. Arch Dis Child 1999;80(suppl 1):A70, G202.

6 Bignardi G. Surveillance of neonatal group B streptococcal infection in Sunderland. Communicable Disease and Public Health 1999;2:64-5. 